Pacific Journal of Mathematics

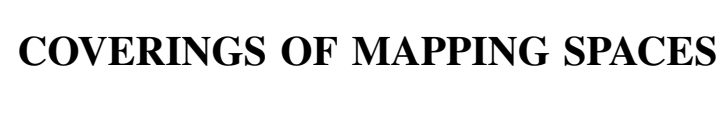




\title{
COVERINGS OF MAPPING SPACES
}

\author{
M. N. Dyer AND A. J. Sieradski
}

The purpose of this paper is to give conditions on a pair of topological spaces $(X, B)$ such that any covering $\rho: E \rightarrow B$ induces a covering map

$$
\bar{\rho}: E^{X} \rightarrow \bar{\rho}\left(E^{X}\right) \subset B^{X}
$$

where $\bar{\rho}(f)=\rho^{\circ} f$ and the mapping spaces have the compactopen topology.

This is given in Theorem 1.1. In the classical theory of (connected) coverings over a space $B$ which is connected, locally pathwise connected and semi-locally 1-connected, it is known that to each subgroup $H \subset \pi_{1}\left(B, b_{0}\right)$ there corresponds a covering projection $\rho: E \rightarrow B$ for which

$$
\rho_{\sharp}\left(\pi_{1}\left(E, e_{0}\right)\right)=H
$$

for some $e_{0} \in \rho^{-1}\left(b_{0}\right)$. Section 2 gives a characterization of those subgroups $H \subset \pi_{1}\left(B^{X}, v\right)$ which correspond to a mapping covering $\rho: E^{X} \rightarrow B^{X}$ for some covering $\rho: E \rightarrow B$. Section 3 gives partial answers to several questions about mapping coverings, such as when mapping coverings are regular or universal.

1. Mapping coverings. Given a topological space $X$ and a map $\rho: E \rightarrow B$, then $\bar{\rho}: E^{X} \rightarrow B^{X}, \bar{\rho}(f)=\rho \circ f$, is continuous if the function spaces of continuous maps $E^{X}$ and $B^{X}$ are each given the compact-open topology. In this section we prove

THEOREM 1.1. Let $\rho: E \rightarrow B$ be a covering projection for which $E$ and $B$ are ANR's, and let $X$ be a compact Hausdorff space. Then $\bar{\rho}: E^{X} \rightarrow B^{X}$ is a covering projection of $E^{X}$ onto $\bar{\rho}\left(E^{X}\right) \subset B^{X}$.

Actually $E$ is automatically an ANR if $B$ is (see $\S 3$ ), so the hypothesis of (1.1) is just a condition on $X$ and $B$. We begin the proof of (1.1) by considering two lemmas, the first of which is a result in Spanier $[6 ; 2.5 .10]$.

LEMMA 1.2. Every Hurewicz fibration with unique path lifting whose base space is locally path connected and semilocally 1-connected and whose total space is locally path connected is a covering projection onto its image.

Since we eventually want to apply this result to the map $\bar{\rho}: E^{X} \rightarrow$ 
$B^{X}$ we need information about the local structure of the function spaces $E^{X}$ and $B^{X}$. If $X$ is a compact metrizable space and $Y$ is an ANR, then the function space $Y^{X}$ is an ANR ([4; p. 186]) and consequently is locally contractible $([4 ; \mathrm{p} .96])$. We now give a direct proof of this local contractibility of $Y^{X}$ which does not require the metrizability restriction on $X$.

Lemma 1.3. If $X$ is a compact Hausdorff space and $Y$ is an ANR, then the function space $Y^{X}$ of continuous maps is locally contractible in the compact-open topology.

Proof. Let the metrizable space $Y$ be considered as a closed subset of a convex set $Z$ in a locally convex topological vector space $L$ ([4; p. 81]). Since $Y$ is an ANR there exists an open neighborhood $W$ of $Y$ in $Z$ together with a retraction $r: W \rightarrow Y$, i.e., $\left.r\right|_{Y}=1_{Y}$.

Given a map $f \in Y^{X}$ and a neighborhood $P$ of $f$, we may assume $P=\bigcap_{i=1}^{n} K\left(C_{i}, U_{i}\right)$ where $K\left(C_{i}, U_{i}\right)=\left\{g \in Y^{x}: g\left(C_{i}\right) \subset U_{i}\right\}$ for each member of the collection $\left\{C_{i}\right\}$ of compact subspaces of $X$ and corresponding member of the collection $\left\{U_{i}\right\}$ of open subsets of $Y$. Since $W$ is an open subset of a convex set $Z$ in a locally convex topological vector space $L$, each open covering $\alpha_{i}=\left\{r^{-1}\left(U_{i}\right), r^{-1}\left(Y-f\left(C_{i}\right)\right)\right\}$ of $W$ admits an open refinement $\beta_{i}$ consisting of convex sets. For each $x \in X$ and index $i$, let $V_{x,}$, be a member of the covering $\beta_{i}$ which contains $f(x)$. Form the convex set $V_{x}=\bigcap_{i=1}^{n} V_{x, i}$ for each $x \in X$ and choose by the regularity of $X$ a closed neighborhood $A_{x} \subset f^{-1}\left(V_{x}\right)$ of $x$. By the compactness of $X$, select points $x_{1}, \cdots, x_{m}$ of $X$ so that $\left\{A_{j}=A_{x_{j}}: j=1, \cdots, m\right\}$ is a collection of closed sets which cover $X$; then let $V_{j}=V_{x_{j}}$ an $V_{j, i}=V_{x_{j}, i}$. Note that

$$
C_{i} \cap A_{j} \neq \varnothing \text { implies } V_{j} \cap Y \subset U_{i} \text {. }
$$

This follows from the facts that $f\left(A_{j}\right) \subset V_{j} \cap Y$ and that $V_{j}$ is contained in $V_{j, i}$, a member of the covering $\beta_{i}$ which refines $\alpha_{i}$.

We define an open neighborhood of

$$
f \in Y^{X} \quad \text { by } \quad K=\bigcap_{j=1}^{m} K\left(A_{j}, V_{j} \cap Y\right) .
$$

Our first claim for $K$ is that it lies in $P$, i.e., that for $g \in K, g\left(C_{i}\right) \subset$ $U_{i}(i=1, \cdots, n)$. Since $\left\{A_{j}\right\}_{j=1}^{m}$ is a cover of $X$, we need merely to show that $g\left(C_{i} \cap A_{j}\right) \subset U_{i}$ for all $i, j$. If $C_{i} \cap A_{j}=\varnothing$, the result holds trivially; if $C_{i} \cap A_{j} \neq \varnothing$, it follows from the relations

$$
g\left(C_{i} \cap A_{j}\right) \subset g\left(A_{j}\right) \subset V_{j} \cap Y \subset U_{i},
$$

the last being due to (1.4). 
Our second claim on $K$ is that it is contractible rel $f$ in $P$. Because $Y$ is contained in $Z$, a convex subset of a topological vector space, we can define a continuous function $H: Y^{X} \times I \times X \rightarrow Z$ by $H(g, t, x)=t f(x)+(1-t) g(x)$. Since on the member $A_{j}$ of the covering $\left\{A_{j}\right\}_{j=1}^{m}$ both $f$ and $g \in K$ take values in the convex subset $V_{j} \subset W$, it follows that $H(K \times I \times X) \subset W$ and therefore the composition $r^{\circ} H$ : $K \times I \times X \rightarrow W \rightarrow Y$ is well defined. The associated map $h: K \times I \rightarrow$ $Y^{X}$ given by $h(g, t)(x)=r(H(g, t, x))$ takes values in $P \subset Y^{X}$ since $r\left(H\left(K \times I \times\left(C_{i} \cap A_{j}\right)\right)\right) \subset r\left(H\left(K \times I \times A_{j}\right)\right) \subset r\left(V_{j}\right)$ and the latter is contained in $r\left(r^{-1}\left(U_{i}\right)\right)=U_{i}$ when $C_{i} \cap A_{j} \neq \varnothing$. Thus $h: K \times I \rightarrow P$ is a homotopy rel $f$ from the inclusion $K \subset P$ to the constant map $K \rightarrow f \in P$. This shows that $Y^{x}$ is locally contractible.

Proof of Theorem 1.1. We first show that if $\rho: E \rightarrow B$ is a covering projection and $X$ is a compact Hausdorff space, then $\bar{\rho}: E^{X} \rightarrow$ $B^{X}$ (and hence $\bar{\rho}: E^{X} \rightarrow \bar{\rho}\left(E^{X}\right)$ ) is a Hurewicz fibration with unique path lifting. For a homotopy $h_{t}: Z \rightarrow B^{X}$ of a map $h_{0}: Z \rightarrow B^{X}$ which lifts to a map $g_{0}: Z \rightarrow E^{X}$, the associated map $h_{t}^{\prime}: Z \times X \rightarrow B$ is a homotopy of the associate $h_{0}^{\prime}: Z \times X \rightarrow B$ which lifts to $g_{0}^{\prime}: Z \times X \rightarrow E$. Since $\rho: E \rightarrow B$ is a Hurewicz fibration, the homotopy $h_{t}^{\prime}$ lifts to a homotopy $g_{t}^{\prime}: Z \times X \rightarrow E$ of $g_{0}^{\prime}$, and therefore the associate $g_{t}: Z \rightarrow E^{X}$ is a homotopy of $g_{0}$ which is a lifting of $h_{t}$. This shows that $\bar{\rho}: E^{X} \rightarrow$ $B^{X}$ is a Hurewicz fibration.

If $\omega, \gamma: I \rightarrow E^{X}$ are paths in $E^{X}$ which cover the same path $\alpha: I \rightarrow B^{X}$ and $\omega(0)=\gamma(0)$, then their associates $\omega^{\prime}, \gamma^{\prime}: I \times X \rightarrow E$ agree on the subspace $0 \times X$ of $I \times X$ and they are liftings of the associate $\alpha^{\prime}: I \times X \rightarrow B$. Since a covering map has the unique lifting property for connected spaces, the fact that $0 \times X$ meets each component of $I \times X$ implies that $\omega^{\prime}=\gamma^{\prime}$ and hence $\omega=\gamma$. This shows that $\bar{\rho}: E^{X} \rightarrow B^{X}$ has unique path lifting.

In view of Lemma 1.2 the proof that $\bar{\rho}: E^{X} \rightarrow \bar{\rho}\left(E^{X}\right)$ is a covering projection is complete once it is shown that $E^{X}$ and $\bar{\rho}\left(E^{X}\right)$ are locally path connected and $\bar{\rho}\left(E^{X}\right)$ is semilocally 1-connected. Since $E^{X}$ is locally contractible by $(1.3)$ the condition on $E^{X}$ is trivial; since $B^{X}$ is also locally contractible the conditions on $\bar{o}\left(E^{X}\right) \subset B^{X}$ follows from the fact that the image of a Hurewicz fibration is the union of path components of the base space.

There are two convenient corollaries of Theorem 1.1. In the first, the notation $\left(Y^{X}\right)_{f}$ is used for the path component of the function space $Y^{X}$ containing $f: X \rightarrow Y$.

Corollary 1.5. If, in addition to the hypotheses of (1.1), $v^{\prime}: X \rightarrow$ $E$ is a lifting of $v: X \rightarrow B$, then $\bar{\rho}:\left(E^{X}\right)_{v^{\prime}} \rightarrow\left(B^{X}\right)_{v}$ is a covering 
projection.

Corollary 1.6. If, in addition to the hypotheses of (1.1), $X$ is locally path connected and $\operatorname{Hom}\left(\pi_{1}\left(X, x_{0}\right), \pi_{1}\left(B, b_{0}\right)\right)=0$ for every $x_{0} \in X$, $b_{0} \in B$, then $\bar{\rho}: E^{X} \rightarrow B^{X}$ is a covering projection.

Corollary 1.5 is immediate. In (1.6) we are asserting that the additional hypotheses imply the surjectivity of $\bar{\rho}: E^{x} \rightarrow B^{X}$. Since $\rho: E \rightarrow B$ is a covering projection a necessary and sufficient condition that a map $f:\left(Y, y_{0}\right) \rightarrow\left(B, b_{0}\right)$ with connected locally path connected domain have a lifting $\left(Y, y_{0}\right) \rightarrow\left(E, e_{0}\right)$ is that in $\pi_{1}\left(B, b_{0}\right), f_{\sharp} \pi_{1}\left(Y, y_{0}\right) \subset$ $\rho_{\ddagger} \pi_{1}\left(E, e_{0}\right)$. Thus the hypothesis Hom $\left(\pi_{1}\left(X, x_{0}\right), \pi_{1}\left(B, b_{0}\right)\right)=0$, for every $x_{0} \in X, b_{0} \in B$, implies that a map $f: X \rightarrow B$ has a lifting on each (path) component of $X$. Because the components of a locally path connected space are open and closed, liftings on the components of $X$ determine a lifting on all of $X$. Thus $\bar{\rho}$ is surjective.

2. Subgroups of $\pi_{1}\left(B^{X}, v\right)$ realizable by mapping coverings. In this section $X$ will always represent a connected finite $C W$ complex of $\operatorname{dim} \leqq n, B$ a path connected simple ANR, and $v: X \rightarrow B$ a selected map. For convenience in stating the main theorem of this section, we define $K_{B}=\operatorname{ker}\left\{r_{\sharp}: \pi_{1}\left(B^{X}, v\right) \rightarrow \pi_{1}\left(B^{X^{\circ}}, r(v)\right)\right\}$, where $r: B^{X} \rightarrow B^{x 0}$ is the map induced by restriction to the 0 -skeleton $X^{0}$ of $X$, and we define $e_{x_{0}}: B^{X} \rightarrow B$ to be the evaluation map $e_{x_{0}}(f)=f\left(x_{0}\right)$ at $x_{0} \in X^{0}$.

If $\rho: E \rightarrow B$ is a covering projection, it follows that $E$ is an ANR (see $\S 3$ ) so that by (1.5) $\bar{\rho}:\left(E^{X}\right)_{v^{\prime}} \rightarrow\left(B^{X}\right)_{v}$ is a covering projection for each lifting $v^{\prime}: X \rightarrow E$ of $v: X \rightarrow B$. We say a subgroup $G \subset \pi_{1}\left(B^{X}, v\right)$ can be realized by a mapping covering if there exists a covering projection $\rho: E \rightarrow B$ with fundamental group $\left(e_{x_{0}}\right)_{\sharp}(G)$ (that is, $\rho_{\ddagger} \pi_{1}\left(E, e_{0}\right)=$ $\left.\left(e_{x_{0}}\right)_{\sharp}(G)\right)$ and a lifting $v^{\prime}: X \rightarrow E$ of $v: X \rightarrow B$ such that the covering projection $\bar{\rho}:\left(E^{X}\right)_{v^{\prime}} \rightarrow\left(B^{X}\right)_{v}$ has fundamental group $G$ (that is, $\bar{\rho}_{\sharp} \pi_{1}\left(E^{X}, v^{\prime}\right)=$ $\left.G \subset \pi_{1}\left(B^{X}, v\right)\right)$. When $v: X \rightarrow B$ is homotopic to the constant map, it follows from $[2 ; 6.1]$ that the condition on the fundamental group of $\rho$ is a consequence of that on the fundamental group of $\bar{o}$.

TheOREM 2.1. A subgroup $G \subset \pi_{1}\left(B^{X}, v\right)$ can be realized by a mapping covering if and only if $G \supset K_{B}$ and $e_{x_{0} \sharp}(G) \supset v_{\sharp}\left(\pi_{1}\left(X, x_{0}\right)\right)$.

Corollary 2.2. When $X$ is simply connected, a subgroup $G \subset$ $\pi_{1}\left(B^{X}, v\right)$ can be realized by a mapping covering if and only if it contains $K_{B}$.

Corollary 2.3. Let $\pi_{1}(B)=0$ for $1<i \leqq n$. Then a subgroup $G \subset \pi_{1}\left(B^{X}, v\right)$ can be realized by a mapping covering if and only if $G \supset K_{B}=H^{n}\left(X ; \pi_{n+1}(B)\right)$ and $\left(e_{x_{0}}\right)_{\sharp}(G) \supset v_{\sharp}\left(\pi_{1}\left(X, x_{0}\right)\right)$. 
ExAmple. Let $X=S^{2}, E=S^{3}, B=P^{3}$, the 3-dimensional real projective space. $P^{3}$ is a topological group $(S O(3)) \Rightarrow P^{3}$ is simple. Let $\rho: S^{3} \rightarrow P^{3}$ be the antipodal identification map. The hypothesis of 2.2 and 2.3 are satisfied for $n=2$. Thus the only subgroups of $\pi_{1}\left(P^{3^{S^{2}}}, v\right)$ realized by a mapping covering are those containing $K_{B}=$ $H^{2}\left(S^{2}, \pi_{3}\left(P^{3}\right)\right) \approx Z$.

Let $c: S^{2} \rightarrow P^{3}$ be the constant map to $p_{0} \in P^{3}$. It follows easily from the spectral sequence in [3] and Theorem 6.1 of [2] that the sequence below is split exact

$$
0 \longrightarrow H^{2}\left(S^{2}: \pi_{3}\left(P^{3}\right)\right) \longrightarrow \pi_{1}\left(P^{3 S^{2}}, c\right) \stackrel{r_{\sharp}}{\longrightarrow} \pi_{1}\left(P^{3}, c\right) \longrightarrow 0
$$

where $H^{2}\left(S^{2} ; \pi_{3}\left(P^{3}\right)\right) \approx K_{p^{3}}$ and $r_{\sharp}$ is induced by the restriction map $r$. $P^{3}$ is a topological group $\Rightarrow P^{3 S^{2}}$ is a topological group $\Rightarrow \pi_{1}\left(P^{3 S^{2}}, c\right)$ is abelian $\Rightarrow \pi_{1}\left(P^{3 . s^{2}}, c\right) \approx Z \oplus Z_{2}$.

Thus the only subgroups of $\pi_{1}\left(P^{3 S^{2}}, c\right)$ which are realizable by a mapping covering are $Z \oplus\{0\}$ and $Z \oplus Z_{2}$ which correspond to $\bar{\rho}: S^{3 S^{2}} \rightarrow$ $P^{3 S^{2}}$ and $I: P^{3 S^{2}} \rightarrow P^{3 S^{2}}$.

We give the proof of (2.1) after a few preliminary propositions. The first involves exact couples of Federer [3] and is easily proved from the data given there.

Proposition 2.4. Let $\rho: W \rightarrow Z$ be a map between path connected simple spaces. Then $\rho$ induces a map

$$
\rho^{i}: \mathscr{C}^{i}(X, W, f) \longrightarrow \mathscr{C}^{i}(X, Z, \rho \circ f)
$$

of the ith Federer exact couples.. Furthermore, there is a commutative diagram

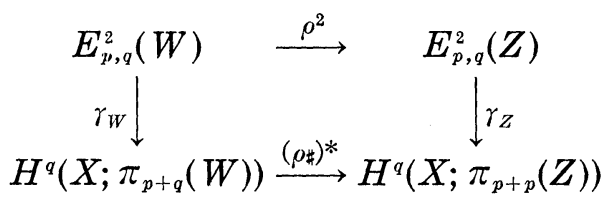

where $\gamma$ is an isomorphism onto if $p>0$ and into if $p=0$.

Proposition 2.5. Let $\rho: W \rightarrow Z$ be a covering projection between path connected simple spaces. Then for the map

$$
\rho^{i}: \mathscr{C}^{i}(X, W, f) \longrightarrow \mathscr{C}^{i}(X, Z, \rho \circ f)
$$

of the i-th Federer exact couple,

$$
\rho^{i}: E_{p, q}^{i}(W) \longrightarrow E_{p, q}^{i}(Z) \quad(i \geqq 2)
$$

is an isomorphism for all $(p, q)$ satisfying either (a) if $p \geqq 1$, then 
$p+q>1$ or (b) if $p=0$, then $q \geqq i$.

Proof. We proceed by induction on $i \geqq 2$. Since $p: W \rightarrow Z$ is a covering projection, $\rho_{\sharp}: \pi_{j}(W) \rightarrow \pi_{j}(Z)$ is an isomorphism for $j \geqq 2$ and a monomorphism for $j=1$. Then in the commutative diagram of (2.4) $\gamma_{W}, \gamma_{Z}$, and $\left(\rho_{\sharp}\right)^{*}$ are isomorphisms for $p+q \geqq 2, p \geqq 1$, hence $\rho^{2}$ is an isomorphism here. For $p=0, q \geqq 2, \gamma_{W}, \gamma_{Z}$ are injective and $\left(\rho_{\sharp}\right)^{*}$ is bijective; consequently $\rho^{2}$ is injective. That $\rho^{2}$ is also surjective when $p=0, q \geqq 2$ follows from the definition of $\mathscr{C}^{1}(X)$ in [3] and the following statement which has the same proof as that of $[6,7.6 .22]$.

(2.6) Let $q \geqq 2$ and let $h: X^{q} \rightarrow Z$ be given such that $h \mid X^{q-1}=$ $p \circ f \mid X^{q-1}$. Then since $\rho_{\sharp}: \pi_{q-1}(W) \rightarrow \pi_{q-1}(Z)$ is injective and $\rho_{\sharp}$ : $\pi_{q}(W) \rightarrow \pi_{q}(Z)$ is surjective, there exists $h^{\prime}: X^{q} \rightarrow E$ such that

$$
h^{\prime}\left|X^{q-1}=f\right| X^{q-1} \text { and } \rho \circ h^{\prime} \cong h\left(\operatorname{rel} X^{q-1}\right) \text {. }
$$

We now assume that (2.5) holds for $i=k-1 \geqq 2$, i.e., $\rho^{k-1}$ is an isomorphism if (a) $p \geqq 1$ and $p+q>1$, or (b) $p=0$ and $q \geqq k-1$. If $p \geqq 1$ and $p+q>1,(q \geqq 0)$, then in

$$
\begin{aligned}
E_{p, q}^{k}(W) & =\frac{\operatorname{ker}\left\{d: E_{p, q}^{k-1}(W) \longrightarrow E_{p-1, q+k-1}^{k-1}(W)\right\}}{\operatorname{im}\left\{d: E_{p+1, q-k+1}^{k-1}(W) \longrightarrow E_{p, q}^{k-1}(W)\right\}} \\
\rho^{k} \downarrow & \\
E_{p, q}^{k}(Z) & =\frac{\operatorname{ker}\left\{d: E_{p, q}^{k-1}(Z) \longrightarrow E_{p-1, q+k-1}^{k-1}(Z)\right\}}{\operatorname{im}\left\{d: E_{p+1, q-k+1}^{k-1}(Z) \longrightarrow E_{p, q}^{k-1}(Z)\right\}}
\end{aligned}
$$

we have $E_{p, q}^{k-1}(W) \approx E_{p, q}^{k-1}(Z)$ by case (a) of the induction hypothesis; $E_{p-1, q+k-1}^{k-1}(W) \approx E_{p-1, q+k-1}^{k-1}(Z)$ when $p \geqq 2$ by case (a) and when $p=1$ by case $(\mathrm{b})$; and $E_{p+1, q-k+1}^{k-1}(W) \approx E_{p+1, q-k+1}^{k-1}(Z)$ because if $q<k-1$ then both are zero, and if $q \geqq k-1$ then case (a) of the induction hypothesis applies. Thus case (a) of (2.5) holds for $\rho^{k}$.

To show that case (b) of (2.5) holds for $\rho^{k}$ suppose that index $p=0$. Here we must show that

$$
\begin{aligned}
& E_{0, q}^{k}(W)=E_{0, q}^{k-1}(W) / \operatorname{im}\left\{d: E_{1, q-k+1}^{k-1}(W) \longrightarrow E_{0, q}^{k-1}(W)\right. \\
& \rho^{k} \downarrow \\
& E_{0, q}^{k}(Z)=E_{0, q}^{k-1}(Z) / \operatorname{im}\left\{d: E_{1, q-k+1}^{k-1}(Z) \longrightarrow E_{0, q}^{k-1}(Z)\right\}
\end{aligned}
$$

is an isomorphism for $q \geqq k$. This is obvious since then $E_{0, q}^{k-1}(W) \approx$ $E_{0, q}^{k-1}(Z)$ by case (b) of the induction hypothesis and $E_{1, q-k+1}^{k-1}(W) \approx$ $E_{1, q-k+1}^{k-1}(Z)$ by case (a).

Before giving the proof of Theorem 2.1, we prove two lemmas.

Lemma 2.7. If $\rho: E \rightarrow B$ is a covering projection with $E$ a path connected simple space and $v^{\prime}: X \rightarrow E$ is a lifting of $v: X \rightarrow B$, then there is a commutative ladder 


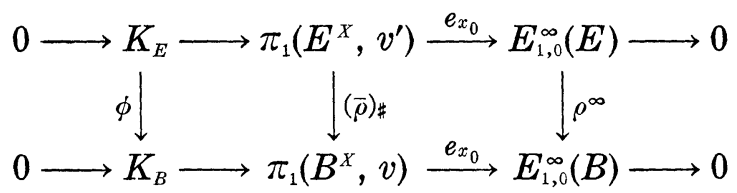

in which the rows are exact and $\phi$ is an isomorphism.

Proof. By a theorem on page 351 of [3], the images of

$$
r_{\sharp}: \pi_{1}\left(E^{X}, v^{\prime}\right) \longrightarrow \pi_{1}\left(E^{X^{0}}, r\left(v^{\prime}\right)\right), r_{\sharp}: \pi_{1}\left(B^{X}, v\right) \longrightarrow \pi_{1}\left(B^{X^{0}}, r(v)\right)
$$

can be identified with the subgroups

$$
\begin{aligned}
E_{1,0}^{\infty}(E) \subset E_{1,0}^{2}(E)= & H^{0}\left(X, \pi_{1}(E)\right)=\pi_{1}(E), \\
E_{1,0}^{\infty}(B) \subset E_{1,0}^{2}(B) & =H^{0}\left(X, \pi_{1}(B)\right)=\pi_{1}(B) .
\end{aligned}
$$

by means of diagonal homomorphisms:

$$
\left\{\begin{array}{l}
\Delta: \pi_{1}\left(E, v^{\prime}\left(x_{0}\right)\right) \longrightarrow \pi_{1}\left(E^{X^{0}}, r\left(v^{\prime}\right)\right) \\
\Delta: \pi_{1}\left(B, v\left(x_{0}\right)\right) \longrightarrow \pi_{1}\left(B^{X^{0}}, r(v)\right)
\end{array}\right\} .
$$

The identification process is natural in $E, B$ and so there is a commutative ladder as indicated.

To show that $\phi$ is an isomorphism we consider the following normal chains (see [3, p. 351]) for $\pi_{1}\left(E^{X}, v^{\prime}\right), \pi_{1}\left(B^{X}, v\right)$ and maps induced by $\bar{\rho}$

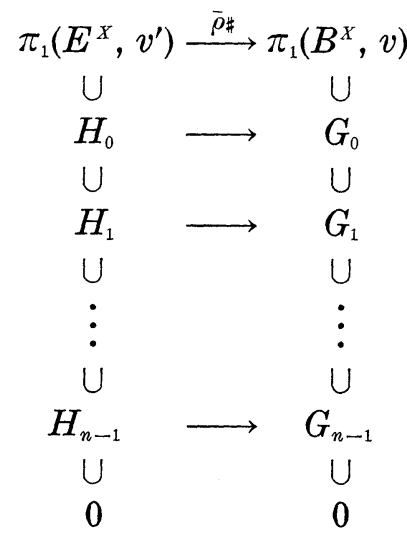

given by

$$
\begin{aligned}
H_{i} & =\operatorname{ker}\left\{r_{\sharp}: \pi_{1}\left(E^{X}, v^{\prime}\right) \longrightarrow \pi_{1}\left(E^{X^{i}}, r\left(v^{\prime}\right)\right)\right\} \\
G_{i} & =\operatorname{ker}\left\{r_{\sharp}: \pi_{1}\left(B^{X}, v\right) \longrightarrow \pi_{1}\left(B^{X^{i}}, r(v)\right)\right\}
\end{aligned} \quad(i=0,1, \cdots, n) .
$$

Thus we must show $K_{E}=H_{0} \approx G_{0}=K_{B}$. By [3, p. 351], there are isomorphisms 


$$
\frac{H_{i}}{H_{i+1}} \approx E_{1, i+1}^{\infty}(E), \frac{G_{i}}{G_{\imath+1}} \approx E_{1, i+1}^{\infty}(B) \quad(i=0, \cdots, n-1)
$$

which can be shown to be compatible with the homomorphisms induced by $\bar{\rho}$. Since $E_{1, i}^{\infty}(E)=E_{1, i}^{k}(E)$ and $E_{1, i}^{\infty}(B)=E_{1, i}^{k}(B)$ for $k>\max (i$, $\operatorname{dim} X-i)$, Proposition (2.5) implies that

$$
\rho^{\infty}: E_{1, i}^{\infty}(E) \longrightarrow E_{1, i}^{\infty}(B)
$$

is an isomorphism for $i \geqq 1$. Via induction and the five lemma, these isomorphisms together with those of (2.9) imply that all but the top homomorphism of the ladder (2.8) are isomorphisms.

Lemma 2.10. Let $\rho: E \rightarrow B$ be a covering projection with $E$ a path connected (simple) space. If in the commutative diagram

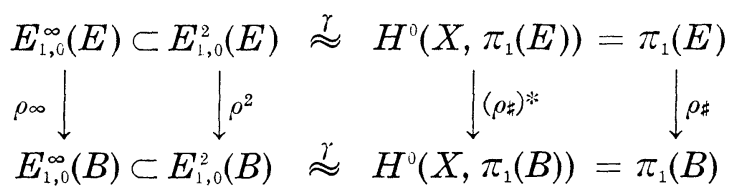

$\rho_{\sharp}\left(\pi_{1}(E)\right) \subset E_{1,0}^{\infty}(B)$, then $E_{1,0}^{\infty}(E)=\pi_{1}(E)$.

Proof. We will show by induction on $k \geqq 2$ that $E_{1,0}^{k}(E)=E_{1,0}^{2}(E)$, or equivalently, that $d_{E}^{k}: E_{1,0}^{k}(E) \rightarrow E_{0, k}^{k}(E)$ is zero. By (2.4) there is a commutative diagram

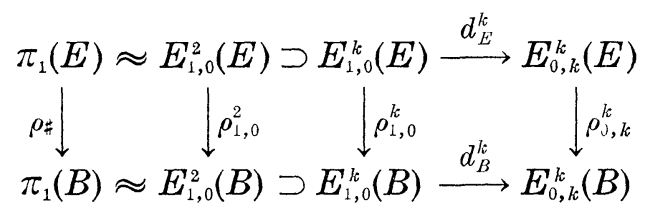

in which $\rho_{0, k}^{k}$ is an isomorphism for $k \geqq 2$ by (2.5) and $\rho_{t, 0}^{k}$ is a monomorphism for $k \geqq 2$ because $\rho_{\ddagger}$ is a monomorphism. In order to prove $d_{E}^{k}$ is zero for $k \geqq 2$, we need only show $\operatorname{ker} d_{B}^{k} \supset \operatorname{im} \rho_{1,0}^{k}$ for $k \geqq 2$. We proceed by induction on $k \geqq 2$. For $k=2$, the statement follows from the relations

$$
\operatorname{ker} d_{B}^{2} \supset E_{1, \mathrm{G}}^{\infty}(B) \supset \rho_{\ddagger}\left(\pi_{1}(E)\right)=\operatorname{im} \rho_{1,0}^{2} \text {. }
$$

If we assume $\operatorname{ker} d_{B}^{j} \supset \operatorname{im} \rho_{1,0}^{j}$ for $j<k, k \geqq 2$, then $E_{1,0}^{2}(E)=E_{1,0}^{k}(E)$ and hence $\rho_{\ddagger}\left(\pi_{1}(E)\right)=\operatorname{im} \rho_{1,0}^{k}$. Then we have the relation

$$
\operatorname{ker} d_{B}^{k} \supset E_{1,0}^{\infty}(B) \supset \rho_{\sharp}\left(\pi_{1}(E)\right)=\operatorname{im} \rho_{1,0}^{k}
$$

which completes the proof by induction.

Proof of Theorem 2.1. Suppose that $G \subset \pi_{1}\left(B^{x}, v\right)$ can be realized 
by a mapping covering, i.e., there exists a covering projection $\rho: E \rightarrow$ $B$ and a lifting $v^{\prime}: X \rightarrow E$ of $v: X \rightarrow B$ such that $\bar{\rho}_{\sharp}\left(\pi_{1}\left(E^{X}, v^{\prime}\right)=G\right.$ and $\rho_{\sharp}\left(\pi_{1}\left(E, v^{\prime}\left(x_{0}\right)\right)=\left(e_{x_{0}}\right)_{\sharp}(G)\right.$. Since we may assume that $E$ is path connected and simple, Lemma 2.7 is applicable. If follows from commutativity of the diagram given there and the surjectivity of $\phi$ that

$$
\bar{\rho}_{\sharp}\left(\pi_{1}\left(E^{X}, v^{\prime}\right)\right) \supset \bar{\rho}_{\sharp}\left(K_{E}\right)=K_{B} \text {, i.e., } G \supset K_{B} \text {. }
$$

Furthermore, we have relations

$$
\left(e_{x_{0}}\right)_{\sharp}(G)=\rho_{\sharp}\left(\pi_{1}\left(E, v^{\prime}\left(x_{0}\right)\right) \supset v_{\sharp}\left(\pi_{1}\left(X, x_{0}\right)\right) .\right.
$$

Conversely, suppose given $G \supset K_{B}$ and $G^{\prime}=\left(e_{x_{0}}\right)_{\sharp}(G) \supset v_{\sharp}\left(\pi_{1}\left(X, x_{0}\right)\right)$. Using the subgroup $G^{\prime} \subset E_{1,0}^{\infty}(B) \subset \pi_{1}\left(B, v\left(x_{0}\right)\right)$, it is possible to construct a covering projection $\rho: E \rightarrow B$ with $E$ a path connected simple space such that $\rho_{\ddagger}\left(\pi_{1}\left(E, e_{0}\right)\right)=G^{\prime}$. Since $v_{\sharp}\left(\pi_{1}\left(X, x_{0}\right)\right) \subset G^{\prime} \subset \rho_{\ddagger}\left(\pi_{1}\left(E, e_{0}\right)\right)$, there exists a lifting $v^{\prime}:\left(X, x_{0}\right) \rightarrow\left(E, e_{0}\right)$ of $v: X \rightarrow B$. Then by Lemma (2.7) there is a commutative diagram

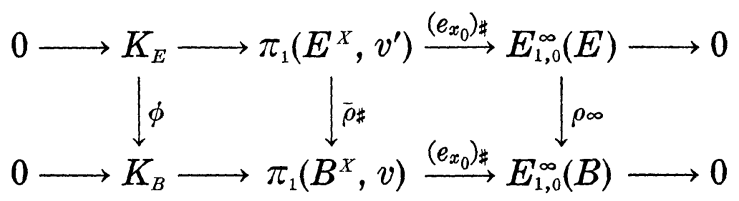

in which $\phi$ is an isomorphism and $\operatorname{im} \rho^{\infty}=G^{\prime}=\left(e_{x_{0}}\right)_{\sharp}(G)$ since $E_{1,0}^{\infty}(E)=$ $\pi_{1}(E)$ by Lemma (2.10). It follows from some diagram chasing that $\bar{\rho}_{\sharp}\left(\pi_{1}\left(E^{X}, v^{\prime}\right)\right)=G$.

3. Miscellaneous questions. Many questions arise concerning mapping coverings. In this section we consider certain ones and give partial answers.

(a) Is a covering space of an ANR an ANR?

(b) If $G$ is a properly discontinuous group of homeomorphisms acting on an $A N R E$, is $E / G$, the orbit space of $G$, an ANR?

(c) If $\rho: E \rightarrow B$ is a covering, what is card $\left(\bar{\rho}^{-1}(f)\right), f \in \bar{\rho}\left(E^{x}\right)$ ?

(d) If $\rho: E \rightarrow B$ is regular, then is $\bar{\rho}: E^{x} \rightarrow B^{x}$ ?

(e) When does a fiber $\bar{\rho}^{-1}(f)$ lie in a single path component of $E^{x}$, i.e., when are all the lifts of $f$ homotopic?

( $f$ ) When is $\bar{\rho}: E^{X} \rightarrow B^{X}$ universal?

For convenience, throughout this section we assume that $B$ is an ANR and $X$ is a compact Hausdorff space.

(a) Since a covering space of an ANR is locally homeomorphic to an ANR, it is an ANR provided it is metrizable (see [4], III, 7.9 and 8.7). So Question (a) now reduces to a consideration of metrizability.

TheOREM 3.1. If $\rho: E \rightarrow B$ is a covering with $B$ metrizable, then $E$ is metrizable. 
The proof utilizes the characterization of $T_{0}$ spaces which are metrizable due to A. H. Stone (see [1], page 196).

COROLlaRY 3.2. Every covering of an ANR is an ANR.

(b) Since $\bar{\rho}: E \rightarrow E / G$ is a covering projection, the question, as in (a), reduces to one of metrizability.

THEOREM 3.3. If a finite group of homeomorphisms $G$ acts on a metric space $E$ without fixed points, then $E / G$ is metrizable.

This again follows from Stone's characterization.

COROLLARY 3.4. G finite, acting without fixed points on an ANR $E \Rightarrow E / G$ is an ANR.

(c) If $X$ is connected and locally pathwise connected, then $f:\left(X, x_{0}\right) \rightarrow\left(B, b_{0}\right)$ has a (unique) lift to $f^{*}:\left(X, x_{0}\right) \rightarrow\left(E, e_{0}\right)$, when $e_{0} \in \rho^{-1}\left(b_{0}\right)$, if $f_{\sharp}\left(\pi_{1}\left(X, x_{0}\right)\right) \subset \rho_{\sharp}\left(\pi_{1}\left(E, e_{0}\right)\right)$. If $E$ is path connected and nonempty, the cardinality of $\bar{\rho}^{-1}(f)\left(f \in \bar{\rho}\left(E^{X}\right)\right)$ reduces to the following question: How many conjugate subgroups of $\rho_{\sharp}\left(\pi_{1}\left(E, e_{0}\right)\right)$ contain $f_{\sharp}\left(\pi_{1}\left(X, x_{0}\right)\right)$ ?

THEOREM 3.5. Let $\rho: E \rightarrow B$ be a regular covering such that $E$ is connected. For any $f \in \bar{\rho}\left(E^{X}\right)$, card $\bar{\rho}^{-1}(f)=\operatorname{card} \rho^{-1}\left(b_{0}\right), b_{0} \in B$.

Proof. $E, B$ ANR $\Rightarrow E, B$ are locally pathwise connected. $E$ is connected $\Rightarrow E, B$ are path connected. $\rho$ is regular $\Rightarrow$ that the group $G$ of covering transformations $\approx \pi_{1}\left(B, \rho\left(e_{0}\right)\right) / \rho_{\sharp}\left(\pi_{1}\left(E, e_{0}\right)\right) \leftrightarrow \rho^{-1}\left(b_{0}\right)$. Then $f \in \bar{\rho}\left(E^{X}\right) \Rightarrow \exists f^{*}: X \rightarrow E \ni f=\rho \circ f^{*}$. Then

$$
\rho^{-1}(f)=\left\{g \circ f^{*} \mid g \in G\right\} \longleftrightarrow \rho^{-1}\left(b_{0}\right)
$$

because $G$ acts transitively on $\rho^{-1}\left(b_{0}\right)$ and any lift of $f$ is determined uniquely by the image of a single point.

With the same hypotheses as 3.5 , we can show that any two path components of $E^{X}$ lying over $\left(B^{X}\right)_{v}$ are homeomorphic. Specifically,

Corollary 3.6. If $v^{\prime}, v^{\prime \prime}$ are any two lifts of $v: X \rightarrow B$, then $\left(E^{X}\right)_{v^{\prime}} \approx\left(E^{X}\right)_{v^{\prime \prime}}$

Proof. $\rho$ regular $\Rightarrow \exists$ a covering transformation $v: E \rightarrow E \ni r_{0} v^{\prime}=$ $v^{\prime \prime}$. Then $\bar{r}: E^{X} \rightarrow E^{X}$ is a covering transformation of $E^{X} \ni \bar{r}\left(v^{\prime}\right)=v^{\prime \prime}$. Thus $\bar{r}:\left(E^{X}\right) v^{\prime} \approx\left(E^{X}\right) v^{\prime \prime}$.

For example, let $X=S^{2}=E$ and $B=P^{2}$, the real projective 
plane. Let $a: S^{2} \rightarrow S^{2}$ denote the antipodal map, $i: S^{2} \rightarrow S^{2}$, the identity. $i \not a$ because $\operatorname{deg}(i)=1$ and $\operatorname{deg}(a)=-1 . \quad \therefore\left(S^{2^{S^{S^{2}}}}\right)_{i} \neq\left(S^{2} S^{2}\right)_{a}$ but if $\rho: S^{2} \rightarrow P^{2}$ is the antipodal identification, then $\rho \circ a=\rho \circ i=\rho: S^{2} \rightarrow P^{2}$. Thus $\left(S^{2 S^{2}}\right)_{a} \approx\left(S^{2^{S^{2}}}\right)_{i}$ as components of $S^{2 S^{2}}$ lying over $\left(P^{2^{S^{2}}}\right)_{\rho}$.

(d) The answer is probably no in general, although the authors have not been able to construct a counterexample. We prove the following

THEOREm 3.7. Let $\rho: E \rightarrow B$ be a covering such that $E, B$ are simple, path-connected ANR's. Let $X$ be a finite $C W$ complex. Then the covering projection

$$
\bar{\rho}: E^{X} \longrightarrow B^{x}
$$

is a regular covering onto $\bar{\rho}\left(E^{X}\right)$.

Proof. As in $\S 2$, the following is a commutative ladder of exact sequences $\ni \bar{\rho}_{\sharp}$ and $\rho^{\infty}$ are injective:

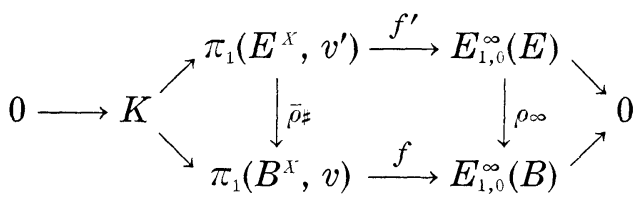

where

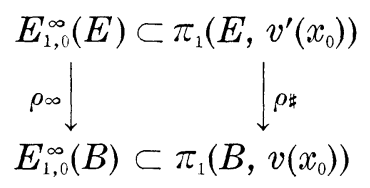

commutes.

$E, B$ are simple $\Rightarrow E_{1,0}^{\infty}(E), E_{1,0}^{\infty}(B)$ are abelian. We will show that $\bar{\rho}_{\sharp}\left(\pi_{1}\left(E^{X}, v^{\prime}\right)\right)$ is a normal subgroup of $\pi_{1}\left(B^{X}, v\right)$ for any $v, v^{\prime} \ni \rho \circ v^{\prime}=v$. Choose $x \in \bar{\rho}_{\sharp}\left(\pi_{1}\left(E^{X}\right)\right), b \in \pi_{1}\left(B^{X}\right)$. Then $f\left(b x b^{-1}\right)=f(b) f(x) f(b)^{-1}=f(x)$ since $E_{i, 0}^{\infty}(B)$ is abelian $\Rightarrow b x b^{-1} x^{-1}=k \in K \Rightarrow b x b^{-1}=k x \in \bar{\rho}_{\#}\left(\pi_{1}\left(E^{X}\right)\right)$.

$$
\therefore \bar{\rho}_{\sharp}\left(\pi_{1}\left(E^{X}, v^{\prime}\right)\right) \triangleleft \pi_{1}\left(B^{X}, v\right) \text { for any } v, v^{\prime} \ni p \circ v^{\prime}=v \text {. }
$$

Theorem 12 on page 74 of $\left.[6] \Rightarrow \bar{\rho}\right|_{{ }_{\left(E^{X}\right)_{v}}}:\left(E^{X}\right)_{v^{\prime}} \rightarrow\left(B^{X}\right)_{v}$ is a regular covering for each $v^{\prime} \in \bar{\rho}^{-1}(v)$. Fix $v^{\prime} \in \bar{\rho}^{-1}(v)$. Suppose $v^{\prime \prime} \in \bar{\rho}^{-1}(v)$ but $\left(E^{X}\right)_{v^{\prime}} \neq\left(E^{X}\right)_{v^{\prime \prime}}$. Then by $3.6 \exists$ a homeomorphism

$$
\bar{r}:\left(E^{X}\right)_{v^{\prime}} \rightarrow\left(E^{X}\right)_{v^{\prime \prime}} \ni \bar{r}\left(v^{\prime}\right)=v^{\prime \prime} \text { and } \bar{\rho} \circ \bar{r}=\bar{\rho} \text {. }
$$

Hence a loop at $v$ in $B^{x}$ lifts to a loop at $v^{\prime}$ if and only if it lifts to a loop at $v^{\prime \prime}$. Therefore $\bar{\rho}: E^{x} \rightarrow \bar{\rho}\left(E^{X}\right)$ is a regular covering. 
(e) We quote a result essentially due to Serre, [5], Proposition 3 , page 479 .

Proposition 3.8. If $G$ is a path-connected, locally path connected, and semilocally 1-connected $H$-space, then each covering transformation on any connected covering space $E$ of $G$ is homotopic to the identity $\operatorname{map} i: E \rightarrow E$.

Corollary 3.9. If $\rho: E \rightarrow B$ is a covering $\ni B$ is an $H$-space, then $\bar{\rho}^{-1}\left(\left(B^{X}\right)_{v}\right)$ is path-connected.

$(f)$ This question only makes sense when we are considering $\left(B^{X}\right)_{v}$. Let us ask: When is $\left(E^{X}\right)_{v}$ a universal covering over $\left(B_{X}\right)_{v}$, where $\rho \circ v^{\prime}=v$ ?

THEOREM 3.10. If $X$ is a $C W$ complex of $\operatorname{dim} \leqq n$ and $E$ is an $n$-connected space, then $\pi_{1}\left(E^{X}, v\right)=0$ for all $v \in E^{x}$.

The proof follows easily from Federer's spectral sequence [3].

\section{BIBLIOGRAPHY}

1. J. Dugundi, Topology, Allyn and Bacon, 1966.

2. M. Dyer, Two term conditions in $\pi$-exact couples, Canad. J. Math. 19 (1967), 1263-1288.

3. H. Federer, A study of function spaces by spectral sequences Trans. Amer. Math. Soc. 82 (1956), 340-361.

4. S. -T. Hu, Theory of retracts, Wayne State Univ. Press, 1965.

5. J. -P. Serre, Homologie singuliere des espaces fibres, Ann. of Math. 54 (1951), 425-505.

6. E. Spanier, Algebraic topology, McGraw-Hill, 1966.

Received January 14, 1969. This research was performed while the authors were supported by a summer research grant at the University of Oregon.

UNIVERSITY OF OREGON

EUGENE, OREGON 


\section{PACIFIC JOURNAL OF MATHEMATICS}

\section{EDITORS}

\author{
H. ROYDEN \\ Stanford University \\ Stanford, California \\ Richard Pierce \\ University of Washington \\ Seattle, Washington 98105
}

\author{
J. DugundJI \\ Department of Mathematics \\ University of Southern California \\ Los Angeles, California 90007 \\ BASIL GoRDON \\ University of California \\ Los Angeles, California 90024
}

\section{ASSOCIATE EDITORS}
E. F. BECKENBACH
B. H. Neumann
F. WOLF
K. YoSHIDA

\section{SUPPORTING INSTITUTIONS}

\author{
UNIVERSITY OF BRITISH COLUMBIA \\ CALIFORNIA INSTITUTE OF TECHNOLOGY \\ UNIVERSITY OF CALIFORNIA \\ MONTANA STATE UNIVERSITY \\ UNIVERSITY OF NEVADA \\ NEW MEXICO STATE UNIVERSITY \\ OREGON STATE UNIVERSITY \\ UNIVERSITY OF OREGON \\ OSAKA UNIVERSITY \\ UNIVERSITY OF SOUTHERN CALIFORNIA
}

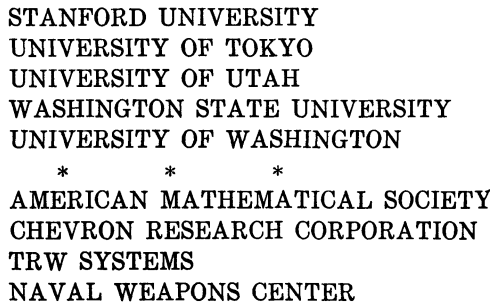

The Supporting Institutions listed above contribute to the cost of publication of this Journal, but they are not owners or publishers and have no responsibility for its content or policies.

Mathematical papers intended for publication in the Pacific Journal of Mathematics should be in typed form or offset-reproduced, double spaced with large margins. Underline Greek letters in red, German in green, and script in blue. The first paragraph or two must be capable of being used separately as a synopsis of the entire paper. It should not contain references to the bibliography. Manuscripts, in duplicate if possible, may be sent to any one of the four editors. Please classify according to the scheme of Math. Rev. 36, 1539-1546. All other communications to the editors should be addressed to the managing editor, Richard Arens, University of California, Los Angeles, California, 90024.

50 reprints are provided free for each article; additional copies may be obtained at cost in multiples of 50 .

The Pacific Journal of Mathematics is published monthly. Effective with Volume 16 the price per volume (3 numbers) is $\$ 8.00$; single issues, $\$ 3.00$. Special price for current issues to individual faculty members of supporting institutions and to individual members of the American Mathematical Society: $\$ 4.00$ per volume; single issues $\$ 1.50$. Back numbers are available.

Subscriptions, orders for back numbers, and changes of address should be sent to Pacific Journal of Mathematics, 103 Highland Boulevard, Berkeley, California, 94708.

PUBLISHED BY PACIFIC JOURNAL OF MATHEMATICS, A NON-PROFIT CORPORATION

Printed at Kokusai Bunken Insatsusha (International Academic Printing Co., Ltd.), 7-17, Fujimi 2-chome, Chiyoda-ku, Tokyo, Japan. 


\section{Pacific Journal of Mathematics}

\section{Vol. 31, No. $2 \quad$ December, 1969}

Efraim Pacillas Armendariz, Quasi-injective modules and stable torsion

classes..........................................

J. Adrian (John) Bondy, On Ulam's conjecture for separable graphs...

Vasily Cateforis and Francis Louis Sandomierski, On commutative rings over which the singular submodule is a direct summand for every module .....

Rafael Van Severen Chacon, Approximation of transformations with continuous

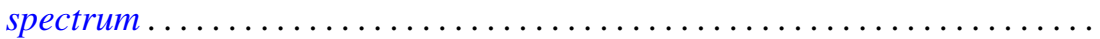

Raymond Frank Dickman and Alan Zame, Functionally compact spaces ...... 303

Ronald George Douglas and Walter Rudin, Approximation by inner

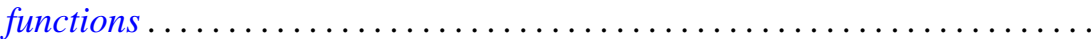

John Walter Duke, A note on the similarity of matrix and its conjugate

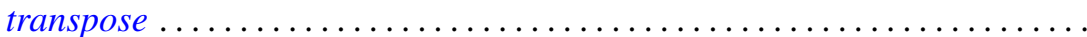

Micheal Neal Dyer and Allan John Sieradski, Coverings of mapping

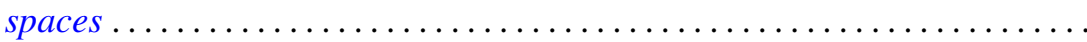

Donald Campbell Dykes, Weakly hypercentral subgroups of finite groups .....

Nancy Dykes, Mappings and realcompact spaces.....................

Edmund H. Feller and Richard Laham Gantos, Completely injective

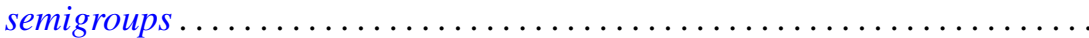

Irving Leonard Glicksberg, Semi-square-summable Fourier-Stieltjes

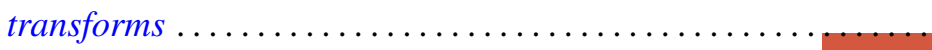

Samuel Irving Goldberg and Kentaro Yano, Integrability of almost cosymplectic structures...

Seymour Haber and Charles Freeman Osgood, On the sum $\sum\langle n \alpha\rangle^{-t}$ and numerical integration ..........................

Sav Roman Harasymiv, Dilations of rapidly decreasing functions ....

William Leonard Harkness and R. Shantaram, Convergence of a sequence of

transformations of distribution functions

Herbert Frederick Kreimer, Jr., A note on the outer Galois theory of rings ...

James Donald Kuelbs, Abstract Wiener spaces and applications to analysis. .

Roland Edwin Larson, Minimal $T_{0}$-spaces and minimal $T_{D}$-spaces...

A. Meir and Ambikeshwar Sharma, On Ilyeff's conjecture .

Isaac Namioka and Robert Ralph Phelps, Tensor products of compact convex sets....

James L. Rovnyak, On the theory of unbounded Toeplitz operators ....

Benjamin L. Schwartz, Infinite self-interchange graphs.......

George Szeto, On the Brauer splitting theorem...

Takayuki Tamura, Semigroups satisfying identity $x y=f(x$,

Kenneth Tolo, Factorizable semigroups .................. 